\title{
Recommended and real-world treatment of knee osteoarthritis in Korea: differences and compromises
}

\author{
Hyun-Sook Kim
}

Division of Rheumatology, Department of Internal Medicine, Soonchunhyang University Seoul Hospital, Seoul, Korea

Received: August 7, 2019

Accepted: August 13, 2019

\section{Correspondence to}

\section{Hyun-Sook Kim. M.D.}

Division of Rheumatology, Department of Internal Medicine, Soonchunhyang University Seoul Hospital, 59 Daesagwan-ro, Yongsan-gu, Seoul 04401, Korea Tel: +82-2-710-3214

Fax: +82-2-709-9554

E-mail : healthyra@schmc.ac.kr https://orcid.org/0000-0001-

9213-7140

\section{See Article on Page 1145-1153}

Knee osteoarthritis (OA) is the major cause of poor quality of life in older adults, and is characterized by degenerative changes of the knee joints that cause pain and impair movement [1]. Treatment guidelines based on expert consensus, in turn based on objective reviews of high-quality meta-analyses, play an important role in defining appropriate treatment and avoiding unnecessary complications [2]. With older age, the average number of comorbid conditions rises, and chronic diseases limiting activity and reducing the efficacy of drug therapy are not uncommon. The recent 2019 Osteoarthritis Research Society International (OARSI) algorithm features patient-centered treatment recommendations for use in clinical practice, and considers various comorbidities in knee OA [3]. The most prevalent and relevant comorbidities in old age are (1) gastrointestinal (GI) comorbidities; (2) cardiovascular (CV) comorbidities; (3) frailty; and (4) widespread pain and/or depression. Oral nonsteroidal anti-inflammatory drugs (NSAIDs) are conditionally recommended for individuals with knee OA without comorbid conditions. NSAIDs of any class are not recommended for patients with high-level CV comorbidities or frailty (Table 1).
In the latest issue of the Korean Journal of Internal Medicine, Park et al. [4] report the treatment patterns of knee OA patients in Korea. They analyzed a large dataset of patients $(n=2,016,516)$ using the knee OA diagnostic code (M17) or any OA diagnostic code (M15 to M19) in combination with a procedure for a knee X-ray in the same claim, and identified patterns of medication use in 2014. Claims data are valuable to clinical epidemiologists; however, although they are readily available on a large scale, they also have important limitations, including a lack of clinical detail and misclassification due to inaccurate coding. The Korean Health Insurance Review and Assessment Service (HIRA) also has the limitation that, as a medication billing system, it is highly likely that information on patients with less severe symptoms who do not need medication are missing. Despite these limitations, the merits of Park et al.'s [4] study include the fact that it evaluates a large nationwide population, including those taking herbal symptomatic slow-acting drugs for osteoarthritis (SYSADOAs), which are commonly prescribed only in Korea.

SYSADOAs are available in many countries as prescription or over-thecounter medications for patients with OA; however, their use remains controversial and guidelines differ. The many SYSADOAs include glucosamine, chon- 


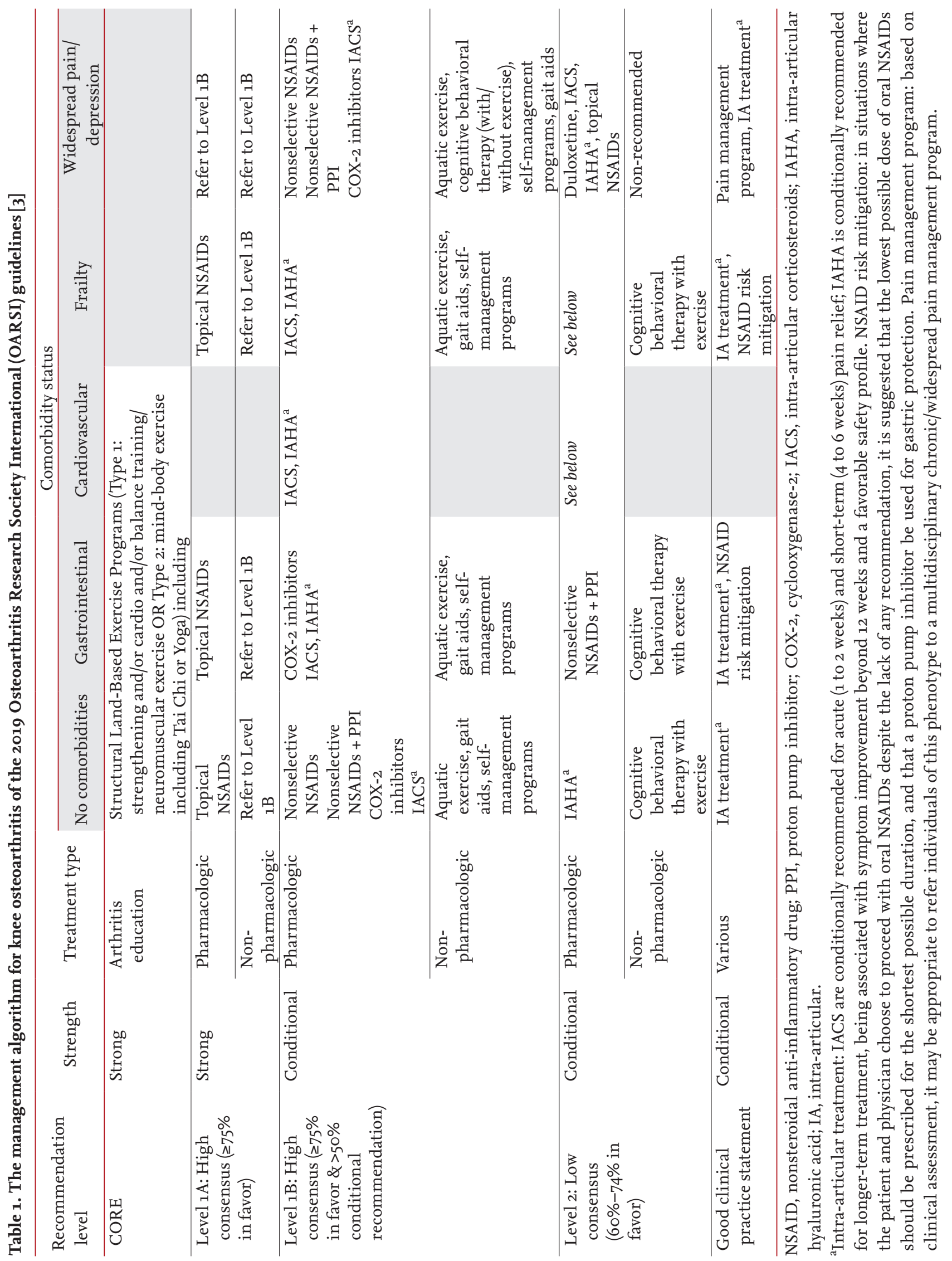


droitin, diacerein, avocado soybean unsaponifiables (ASU) and herbal SYSADOAs which are mixed extracts of various herbs [5]. The European Society for Clinical and Economic Aspects of Osteoporosis, Osteoarthritis, and Musculoskeletal Diseases (ESCEO) published recommendations for the management of knee OA in 2019, in the form of a stepwise treatment algorithm to guide physicians [6]. The ESCEO comprehensively summarized the safety data and commissioned several meta-analyses on the safety of different classes of anti-OA medications, including SYSADOAs and cyclooxygenase-2 (COX-2) inhibitors. Ultimately, the ESCEO strongly recommended that pharmaceutical-grade crystalline glucosamine sulfate and chondroitin sulfate should serve as step 1 background SYSADOAs, and that other SYSADOAs (diacerein and ASU) may be used as alternative step 1 background therapies. The 2019 OARSI guidelines do not mention SYSADOAs, instead emphasizing core treatments, structured land-based exercise programs, dietary weight management in combination with exercise, and mind-body exercises in knee OA treatment (Table 1). Additionally, acetaminophen/paracetamol (AAP), which was previously regarded as a first-line pharmacological treatment for $\mathrm{OA}$, was not recommended regardless of comorbidity status. In the study of Park et al. [4], SYSADOAs (43.4\%) and AAP-containing analgesics (31.2\%) were frequently used at all times during follow-up. Of the SYSADOAs, diacerein (16.6\%) and herbal SYSADOA A (16.1\%) were the most commonly employed, followed by ASU (8.2\%) and herbal SYSADOA B (7.9\%). The overall percentage of total herbal SYSADOA use was as high as $29.7 \%$.

The results of Park et al. [4] revealed unique prescription patterns, including common use of NSAIDs (82.5\%), SYSADOAs (43.4\%), and oral corticosteroids (CSs) (8.6\%). Although, NSAIDs are widely employed because they are highly effective for controlling OA pain, they are clearly associated with GI and CV complications. Selective COX-2 inhibitors and nonselective NSAIDs in combination with proton pump inhibitors were conditionally recommended because they relieve pain and improve functional outcomes in knee OA patients with GI comorbidities, and have more favorable upper GI safety profiles than non-selective NSAIDs alone in 2019 OARSI guidelines [3]. Topical NSAIDs were strongly preferred to oral analgesics for knee OA patients with no comorbidities, GI or CV comorbidities, and for frail pa- tients. Even after excluding specific comorbidities, such as chronic obstructive pulmonary disease, asthma, and undifferentiated inflammatory arthritis (all of which are managed with CS), the prevalence of CS use in knee OA patients was extremely high in Park's study. Further work is needed to explore why CS use is so high and to rule out abuse thereof.

In clinical practice, intra-articular corticosteroids (IACS) are widely considered to be the most effective nonsurgical treatment for acute knee OA, providing short-term pain relief and substantial functional improvement [7]. Park et al. [4] reported that the prevalence of IACS use was very low (0.18\%), but patients who received IACS were older and had more comorbidities. The 2019 OARSI guidelines conditionally recommend the use of IACS and intra-articular hyaluronic acid (IAHA) to treat knee OA for all patient groups. A "Good clinical practice" statement pertaining to intra-articular treatments for all comorbidity subgroups emphasized that IACS can provide short-term pain relief(Table 1) [3].

Currently, considerable emphasis is being placed on the need for a patient-centered approach for knee OA considering individual comorbidities. Rigorous evidence supporting recent management guidelines for knee OA treatment in Korea is lacking. Further detailed exploration of real-world knee OA treatment patterns in patients with various comorbidities is essential. Given the differences between international recommendations and the actual situation in Korea, clinical guidelines that recognize current drug therapy patterns are needed; only then will it be possible to make improvements.

\section{Conflict of interest}

No potential conflict of interest relevant to this article was reported.

\section{REFERENCES}

1. McAlindon TE, Bannuru RR, Sullivan MC, et al. OARSI guidelines for the non-surgical management of knee osteoarthritis. Osteoarthritis Cartilage 2014;22:363-388.

2. Loeser RF. Aging processes and the development of osteoarthritis. Curr Opin Rheumatol 2013;25:108-113.

3. Bannuru RR, Osani MC, Vaysbrot EE, et al. OARSI guide- 
lines for the non-surgical management of knee, hip, and polyarticular osteoarthritis. Osteoarthritis Cartilage 2019 Jul 3 [Epub]. https://doi.org/10.1016/j.joca.2019.o6.o11.

4. Park HR, Cho SK, Im SG, et al. Treatment patterns of knee osteoarthritis patients in Korea. Korean J Intern Med 2019;34:1145-1153.

5. Honvo G, Reginster JY, Rabenda V, et al. Safety of symptomatic slow-acting drugs for osteoarthritis: outcomes of a systematic review and meta-analysis. Drugs Aging
2019;36(Suppl 1):65-99.

6. Kucharz EJ, Szanto S, Ivanova Goycheva M, et al. Endorsement by Central European experts of the revised ESCEO algorithm for the management of knee osteoarthritis. Rheumatol Int 2019;39:1117-112 3.

7. Zeng C, Lane NE, Hunter DJ, et al. Intra-articular corticosteroids and the risk of knee osteoarthritis progression: results from the osteoarthritis initiative. Osteoarthritis Cartilage 2019;27:855-862. 